\begin{tabular}{|c|c|c|c|}
\hline Change of $\mathrm{I}$ & $\begin{array}{r}T \\
\text { ee Energ }\end{array}$ & $\begin{array}{l}\text { V. } \\
\text { Heat of Mixing }\end{array}$ & \\
\hline $\begin{array}{c}\text { Molar fraction of benzene. } \\
x \text {. }\end{array}$ & $A_{\text {calorie. }}$ & $\begin{array}{l}\text { Feat of mixing } \\
\text { q. Caloric. }\end{array}$ & $Q / A$ \\
\hline 0.0975 & 25.4 & 54.2 & $2 \cdot I 3$ \\
\hline 0.1616 & 37.8 & $8 I .1$ & 2.15 \\
\hline $0.274 \mathrm{I}$ & 52.7 & III.Z & 2. $\mathrm{YI}$ \\
\hline $0.377 \mathrm{r}$ & $6 \mathrm{x} .0$ & 129.2 & 2.12 \\
\hline 0.4776 & 64.0 & 134.9 & 2. II \\
\hline 0.7980 & $38 . \mathrm{r}$ & 80.8 & 2.12 \\
\hline
\end{tabular}

The values of $A$ in second column have been obtained by interpolation on the $x-A$ curve which is constructed by means of the data given in Table II. The third column is the value of $q$, the heat of mixing for one mol of solution at $25^{\circ}$ obtained by $\mathrm{H}$. Hirobe. ${ }^{1}$ It is to be observed that the ratio $q / A$ is constant for all values of $x$ as shown in the fourth column. It appears, then, that there is a comparatively simple relation between heat of mixing and loss of free energy. Regretfully, however, I was not able to continue this study longer, and could not decide the general relation between them.

I express my heartfelt thanks to Professor $\mathrm{K}$. Ikeda for his kind guidance during this investigation.

TORYO, JAPAN.

[Contribution from the Departuent of Chemistry of Princeton University.]

\title{
A STUDY OF THE OCCLUSION OF HYDROGEN AND OXYGEN BY METAL ELECTRODES.
}

By Earle A. HaRding and Donaid P. SMitg.

Received June 26, 1918.

The purpose of the present study was to confirm and extend observations recently reported ${ }^{2}$ upon the changes of electrical resistance which result from the cathodic occlusion of hydrogen by small palladium wires. The most important observation of the previous investigation was a continued increase in the electrical resistance of palladium-hydrogen after electrolysis had been interrupted, and a relation between the current strength and the steady value of resistance attained during electrolysis. In the present experiments the observations upon the changes produced in the electrical resistance of palladium wires and ribbons have been supplemented by measurements of the accompanying changes of length, by studies upon the corresponding effects of oxygen occlusion, and by some similar experiments upon wires of platinum, tantalum, and iron.

\section{Experiments with Palladium.}

The experiments upon palladium, which constitute the greater part of those to be described, involved simultaneous measurements of electrical

1 The paper has not yet been published.

2 This Journal, 38, 2577 (I9I6). 
resistance and of length, and were conducted in the cell shown in Fig. I. The details of construction of this cell may be seen from the figure. Its arrangement was essentially such that the wire $c d$, which formed the object of the experiment, was held between the fixed pair of platinum conductors $l^{\prime}, m^{\prime}$ and the suspended conductors $l, m$. The latter, together with the glass tube $g$, in which they were contained, depended by means of the ring $r$, from a stout wire hook upon the bottom of a steel millimeter scale. The scale in turn was suspended in a vertical position by two silk cords, hung from parallel grooves in a brass quadrant which formed the end of one arm of a small sensitive balance. Thus the experiment wire was kept fully extended with the minimum of tension, and every change in its length produced a corresponding displacement of the millimeter scale. These displacements were observed, in relation to a fixed point of fine platinum wire, by means of a reading telescope, which was supported upon a stone base, and was provided with a micrometer scale, ruled in roo divisions, which replaced the ordinary cross-hairs. The telescope was so adjusted that the one hundred divisions of the micrometer covered nineteen divisions of the steel scale, thus forming a modified vernier. By this means it was possible to read changes in length with a precision of $0.01 \mathrm{~mm}$., and an accuracy of not less than $0.02 \mathrm{~mm}$.

The conductors $l, m, l^{\prime}, m^{\prime}$ led to the arms of a Kelvin double bridge, through the heavy copper conductors $w, x, y, z$, which formed at the same

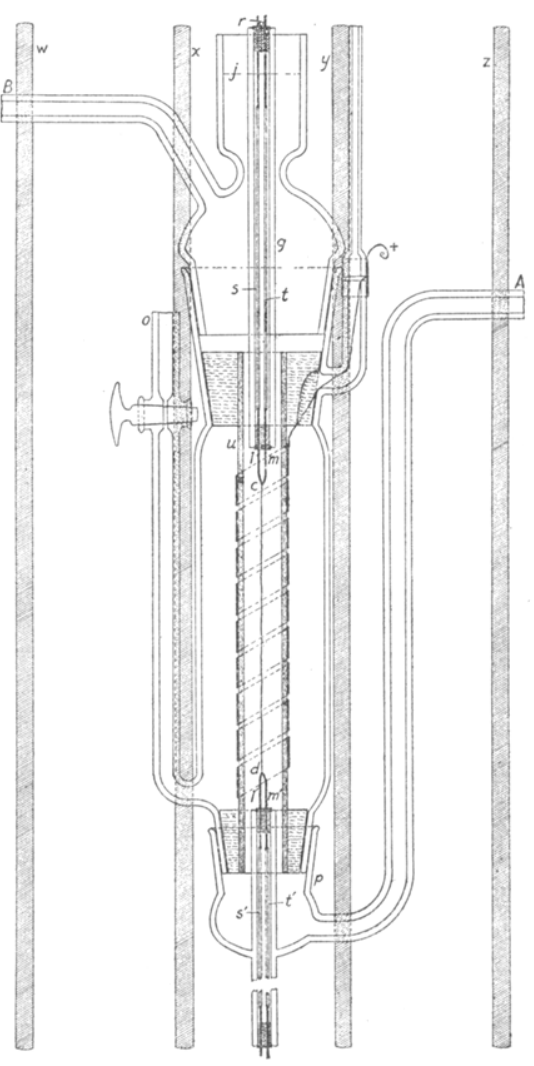

Fig. I. time a framework for the support of the cell. The flexible leads necessary were composed of cables of fine silver wire. All connections were made by soldering, and by employing silver wires of $\mathrm{I} .3 \mathrm{~mm}$. for the intermediate portions $s, t$. Thus the potential leads were given resistances as small and as nearly alike as possible. The Kelvin bridge used, composed of a dial resistance box, and separate ratio coils, gave measurements accurate to about one part in six thousand. For certain experiments described later, a more accurate bridge was substituted. 
In order to introduce a new experiment wire, the cell was taken apart by removing the ground glass cap $p$. This permitted the upper glass tube $g$, carrying the flexible leads $l, m$, to be drawn downward through the porous procelain tube $u$. The cathode wire was then fused to the leads. In carrying out this operation much difficulty was at first experienced in fusing small palladium wires to the large platinum leads. The method finally adopted consisted in hammering the platinum conductors into the form of a thin spade, to which the palladium wire could be fused without much difficulty. In the case of $0.05 \mathrm{~mm}$. wires, it was found better to dip the platinum spade, with the palladium wire folded over the edge, into a small globule of molten gold.

The cell was secured, by cords covered with shellac, to a rigid framework composed of the 4 copper conductors $w, x, y, z$, held in place by heavy glass rods and a metal and wood base. This entire part of the apparatus was placed in a large oil thermostat which maintained a temperature of $25.00 \pm 0.01^{\circ}$.

Before the introduction of the electrolyte the experiment wire was in every instance annealed to constant resistivity; in general, this was done after the air had been displaced from the cell by nitrogen. Commercial tank nitrogen was employed, which had been purified by means of ammoniacal copper carbonate and copper, in an apparatus similar to that described by Van Brunt. ${ }^{1} \mathrm{~A}$ few experiments in which the annealing was made in air will be discussed later. The current used in annealing, which was introduced through the bridge leads, sufficed to bring the wires to a dull red heat. In order to avoid endangering the surrounding glass portions of the cell, the current was applied only for periods of ro seconds with intervals of like duration. After thus annealing for 5 minutes the operation was interrupted and observations of the electrical resistance of the wire were taken until constancy had been attained. This series of operations was repeated until the resistivity of the wire was reproduced from one annealing to the next, to within about one part in five hundred.

When wires which were already charged with hydrogen were subjected to annealing, in preparation for a new experiment, it was found necessary to raise the temperature with special care, since otherwise the wires were very likely to be ruptured, or to be so weakened as to give way during the subsequent treatment. This was undoubtedly due to the fact that the hydrogen is given off with great suddenness when the temperature is raised to a certain value. If measured currents of successively greater magnitudes are passed through a wire saturated with hydrogen, no perceptible alteration either in resistance or in length is produced, beyond the very gradual change which occurs spontaneously at ordinary tempera-

1 This JovrNas, 36, 1448 (19t4). 
tures, until the intensity of the current is about one-third of that which is required to raise the wire to redness. If the current is stepped up by small increments, the evolution of the gas begins with great rapidity when the critical point has been reached. At this current most of the gas can be expelled, and resistance and length restored very nearly to the values which they possessed before hydrogen had been occluded. The last portions of the hydrogen, however, are driven off only at a higher temperature. The current was, therefore, in every instance increased gradually until the wire reached a dull red heat. By annealing some time at this temperature both resistance and length were brought to constancy. The values thus reached differed somewhat from the values before the occlusion but these differences were apparently due wholly to changes in the length; for the resistivities, calculated upon the assumption that the changes of the cross-section of the wire were negligible, returned very closely to their original values.

Thus in the case of the wire $\mathrm{C}_{2}$, the resistivity obtained after annealing the new wire was $\sigma \times 10^{4}=0.1347$, while 3 subsequent annealings, made after successive saturations with hydrogen, gave 0.1348 , o. I347, and 0.1337 . Similarly in the case of wire $\mathrm{C}_{5}$, the original value was o. I390, and that obtained after 3 runs was 0. I39I. It may be said, therefore, that electrolytic treatment, and subsequent annealing conducted as has been described, produce scarcely any permanent change in the resistivity of a palladium wire. This is of importance in view of the very marked alteration caused in the metal when hydrogen is expelled from palladium by rapid heating in vacuo, as was done by Graham ${ }^{1}$ and by numerous later investigators.

After the experiment wire had been annealed, the outer or anode chamber of the cell was filled through the side tube $o$. Electrolyte was also admitted to the cathode chamber, and allowed to circulate through the latter at the rate of about 4 liters every 24 hours, during the entire course of an experiment. In order that this circulation might be maintained, and the level of the electrolyte kept constant at the point $j$, the liquid was allowed to flow from an upper reservoir arranged upon the principle of the Marriott bottle. From this it passed through a glass coil within the thermostat, entered the cell at $A$, and leaving at $B$, was received by a second reservoir. With this open type of cell, necessitated by the measurement of the changes of length of the cathode wire, it was not possible wholly to exclude dissolved air from the electrolyte. The presence of traces of oxygen was, however, without material influence upon any of the phenomena observed, except that it affected the form of the "evolution curve," as will be mentioned later.

The system of tubes conducting the electrolyte to and from the cathode

1 Proc. Roy. Soc. (London), 17, 2 I2 (1869). 
chamber was provided with cocks so that the cell could be isolated from the remainder of the apparatus. This permitted the acid to be removed in preparation for re-annealing the cathode wire, and the cell to be washed out. The washing was always continued until a test for sulfate was no longer obtained. The electrolyte used throughout the entire course of these experiments was $2 N$ sulfuric acid, purified and diluted as described in a paper already cited. ${ }^{1}$

The palladium employed was of two qualities. The first of these, designated $\mathrm{C}$ in the tables which follow, was commercial metal found to have a resistivity which varied from wire to wire, between about $\sigma \times 10^{4}=$ 0.1347 and 0.1376 at $25^{\circ}$. The other palladium, designated $P$, had been carefully purified, being of the lot referred to by Martin. ${ }^{1}$ Its resistivity was about 0 . 1099 at $25^{\circ}$. The values for commercial and for pure palladium calculated for this temperature from the data of Barrett ${ }^{2}$ would be 0.1795 and 0.1079 , respectively.

The very steady electrolytic current, furnished by Daniell cells, was measured with a milliammeter in most of the experiments; but in those the results of which are shown in Table I, it was observed by a fall-ofpotential method, employing a potentiometer and an excellent resistance box. In these latter instances the current measurements were accurate to one part in ten thousand, or better.

\section{Changes of Resistance of Palladium.}

A typical complete experiment upon palladium consisted of 4 parts, corresponding to the 4 branches of the curve shown in Fig. 2, which is an ideal representation, and not drawn from the results of a single experiment.

During the first part, represented by the branch A B C, or "charging curve," hydrogen was electrolytically evolved upon the palladium cathode and occluded by the latter; in the second part, represented by the "evolution curve" CDE, hydrogen was allowed to escape spontaneously from the cathode, after the interruption of electrolysis; in the third part which gives the "renewal curve" EF G H I, the cathode from which hydrogen had partly escaped, was again caused to occlude as a result of renewed electrolysis; and in the fourth part, corresponding to the "discharge curve" I J K, the wire was freed from hydrogen by electrolysis with the direction of the current reversed.

Resistance curves, and not resistivity curves, are given in the present paper. It may be mentioned, however, that curves of every type have also been plotted in terms of resistivity. The general form of the curves was the same, showing that changes in the length of the wires were not responsible for the variations observed.

1 This Jolrnal, 38, 2577 (I916).

2 Proc. Phys. Soc. (London), 26, 347 (Ig14). 
The first two curves, just designated "charging curve" and "evolution curve" are closely related and may best be discussed together.

The supplementary increase of resistance after the interruption of electrolysis, represented by the evolution curve, occurs not only when charging has been continued to the attainment of a steady value of resistance, as is represented by C DE in Fig. 2, but also takes place when the interruption is made during the earlier stages of electrolysis (see $\mathrm{M} \mathrm{NO}$ and $P Q R$, Fig. 2). Hence, when the electrolytic current is momentarily broken to make possible the observation of electrical resistance, from which the position of any point upon the charging curve is determined,

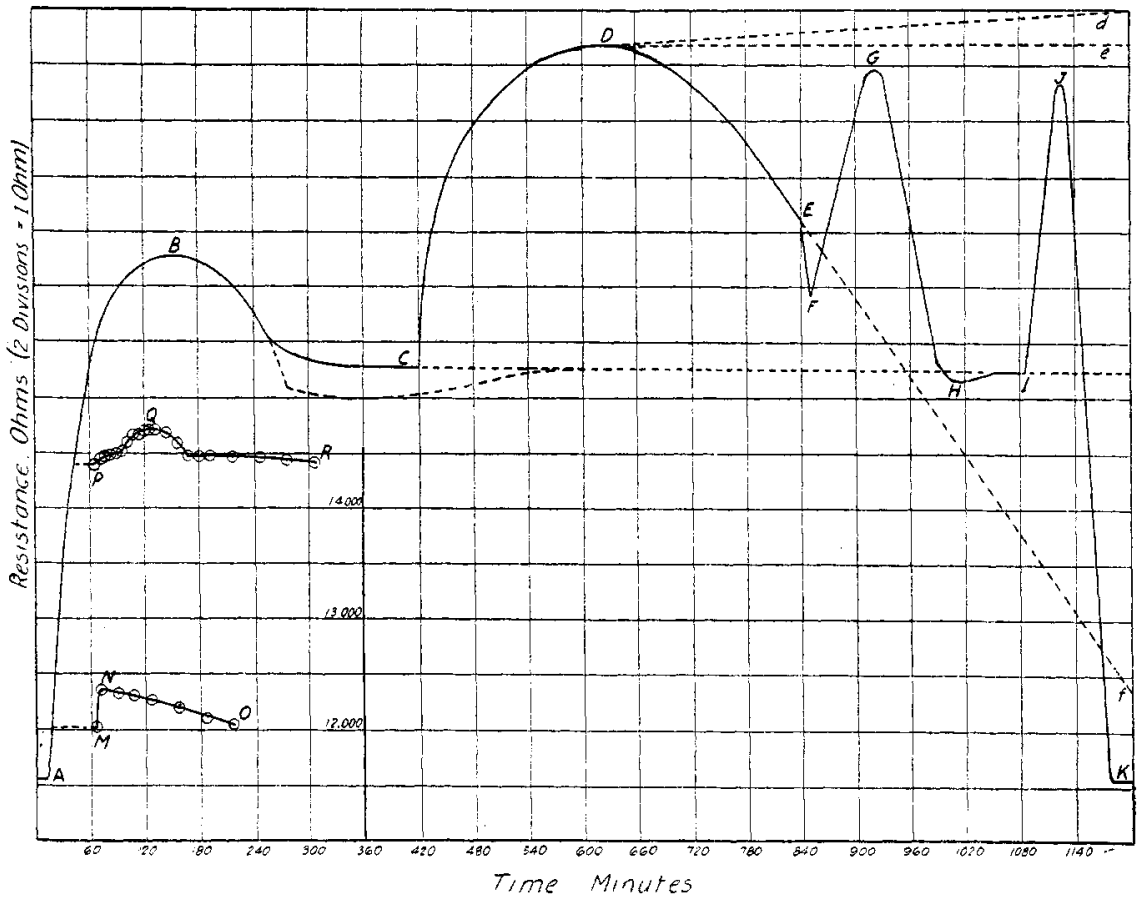

Fig. 2.-Typical experiment.

an accelerated increase of resistance begins at once. As a consequence, the resistance values observed during charging are somewhat higher than the true values, which would be obtained if it were possible to make measurements while the electrolysis was actually proceeding.

Some attempts to make these measurements, without interrupting electrolysis, by the employment of an alternating current bridge were unsuccessful, owing to the exceptionally difficult problem of measuring a varying resistance, accompanied by a varying electromotive force within the circuit and a varying electrostatic capacity. Since, therefore, it was necessary to interrupt the electrolysis during each resistance measure- 
ment, it seemed desirable to vary the periods of interruption, in order to make sure that the error produced was not sufficient to cause the observed charging curve to differ materially from that which would be obtained under ideal conditions. The variation was secured by employing two different methods of measurement. In the first of these, the electrolysis was momentarily interrupted merely by opening a switch; in the second, much briefer interruptions were secured by means of a rotating commutator, constructed for the purpose. The agreement between the curves obtained with interruptions of ro seconds or more, in the first method, and those obtained with interruptions of approximately o. I second, given by the commutator, show that at least the general form is unaffected by an error of this kind. Compare curves Nos. I, 2 and 3, with No. 4 in Fig. 3.

From Martin's experiments it appeared that the charging curve rises regularly to a steady value; and it also seemed that the magnitude of the supplementary rise, shown by the evolution curve, depends upon the size of the wire. In those experiments the highest current density employed was one of 0.32 amp. per sq. dcm., while in most instances the value was much smaller. A more thorough examination of the influence of this factor, which has been made in the present experiments, has shown it to be of the first importance. With current densities somewhat higher

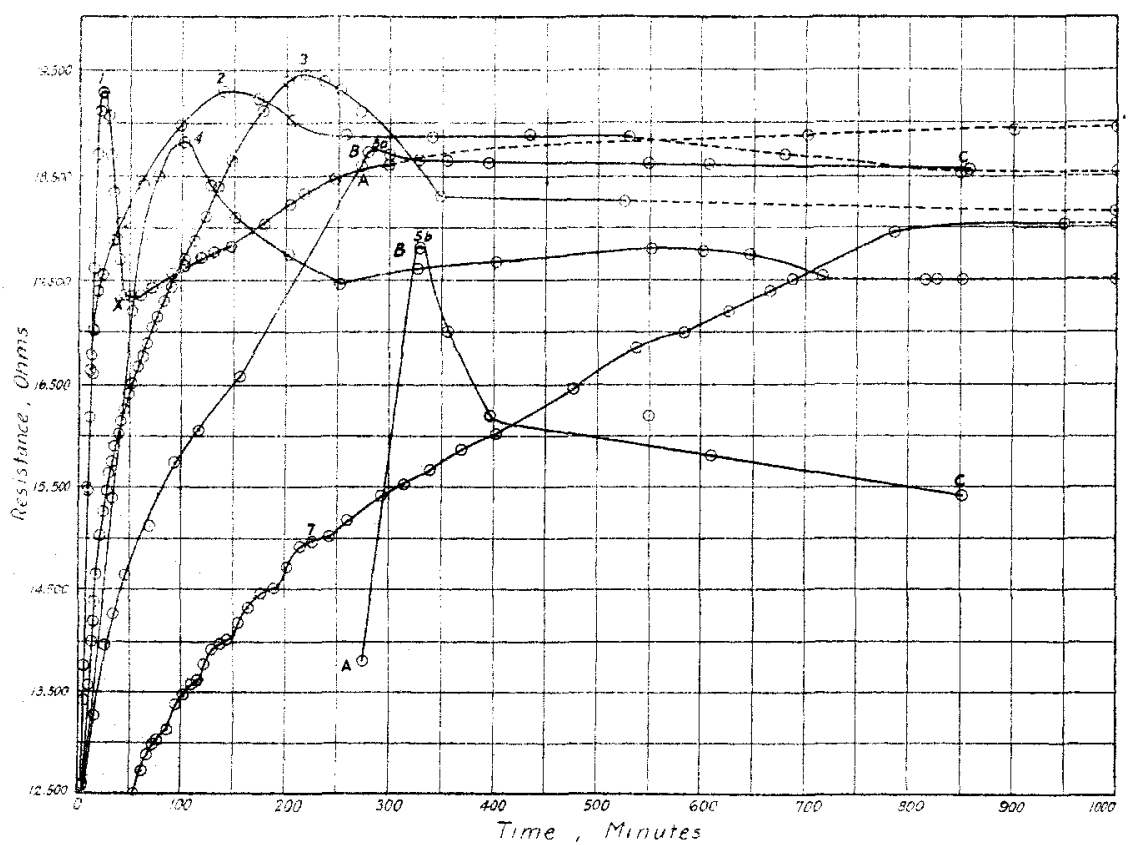

Fig. 3.-Charging curves (No. 7.-Changes of length for curve No. 3). Ordinates, $\mathrm{I}$ div. $=0.2 \mathrm{~mm}$. 
than those previously employed, the charging curve passes through an unmistakable maximum before coming to the steady value, and a reëxamination of Martin's data shows that this maximum is perceptible in that particular one of his experiments in which the highest current density was used. These facts are made plain by Fig. 3, in which the experiments of the present series are represented by curves Nos. I-4, while Martin's is shown in No. $5^{a}$, and the critical portion of the last is given on a larger scale by No. $5^{b}$.

The current density also determines the rapidity with which the resistance, during electrolysis, reaches a steady value, and hence, the steepness of both the rising and falling branches of the charging curve. This fact is shown by Curve No. I, Fig. 3, and is evidenced by the results of all the present experiments on palladium, which were 34 in number.

Moreover, the steady value reached by the resistance ( $\mathrm{C}$ in Fig. 2) is not dependent upon the size of the wire, as seemed to be indicated by Martin's experiments, but is wholly determined, at least within very narrow limits, by the current density. This will be apparent from the results shown in Table $I$, taken from experiments in which the current was measured to within $2 \times \mathrm{ro}^{-7}$ amp., and by unremitting attention was kept constant within very nearly the same limits. The current densities, although thus maintained with great constancy, are known only to within about $4 \%$, owing to the error in the determination of the diameter of the small wire cathode. In this table the first column shows the quality of the palladium, together with the number of the wire and of the experiment. The second column gives the diameter of the wire. In the third column the current densities are shown, reduced to amperes per sq. dcm. The headings of the remaining columns are self-explanatory.

\section{TABLE I.}

$\begin{array}{cccccc}\text { Expt. } & \text { Diam. of wire. } & \text { C. D. } & \begin{array}{c}\text { \% increase over the original resistance. } \\ \text { value. }\end{array} & \begin{array}{c}\text { Maximum } \\ \text { value. }\end{array} & \begin{array}{c}\text { During } \\ \text { evolution. }\end{array} \\ \text { C } 6,5 & 0.05 & \text { I } .73 & 42.2 & 70.8 & 28.6 \\ \text { C } 6,6 & 0.05 & 1.73 & 37.3 & 71.7 & 34.4 \\ \text { C } 7,1 & 0.10 & 1.73 & 40.9 & 69.2 & 28.3 \\ \text { C } 7,2^{7,3} & 0.10 & 3.81 & 35.5 & 68.5 & 33.0 \\ \text { C } 7,3 & 0.10 & 0.018 & 68.5 & 69.6 & 1.1\end{array}$

From these results it will be seen that 3 experiments were carried out with the same current density, two of them upon a $0.05 \mathrm{~mm}$. wire, and one on a wire of 0 . Io $\mathrm{mm}$. diameter. These gave, as a mean value, $40.1 \%$ increase over the original resistance. Owing to the uncertainty, already discussed, which is inherent in all the observations taken during electrolysis, the variations are probably merely accidental.

This influence of current density is also accountable for the inverse 
response of resistance to current, observed by Martin (loc. cit., Fig. 3), and shown also in the lower curve of Fig. $4 b$ of this paper. This will be further discussed in connection with the changes of length.

The evolution curve was observed in all of the present experiments and evidence was found, in addition to that already obtained by Martin, that the increase of resistance after the interruption of electrolysis is due to changes within the wire, and not to any effect in the surrounding electrolyte. This evidence is afforded by the following experiment: after a steady value in the electrical resistance had been reached, the electrolyte was withdrawn from the cell and the latter was washed out with distilled water until no test for a sulfate could be obtained. The resistance continued to rise during this treatment and at the time when measurements were suspended, had reached a value of $20.175 \mathrm{ohms}$, as compared with an average value of 20.253 ohms for 7 experiments on the same wire, made while it was surrounded by electrolyte. It may be supposed that the average value would have been reached also in this experiment without electrolyte, if sufficient time had been allowed for the attainment of the maximum.

The magnitude of the supplementary gain in resistance represented in the evolution curve is evidently simply the difference between the maximum value reached ( $D$ in Fig. 2), and the steady value ( $C$ in Fig. 2), at which the evolution begins. It has already been shown that the latter of these is dependent upon the current density. The maximum value $D$, on the other hand, although probably slightly altered by changes in the current, is very little affected, as may be seen from the fifth column of Table I. As a consequence the difference between the two values $\mathrm{C}$ and $\mathrm{D}$, which constitutes the magnitude of the supplementary gain in resistance, shown in the evolution curve, is determined entirely by the steady value $C$, and the supplementary gain is greater, the greater the current density at which the resistance is allowed to reach the steady value. This relation between current density and supplementary gain of resistance may be seen in Table I, Cols. 3 and 6.

The supplementary gain of resistance is not strikingly noticeable with current densities of less than about $0.3 \mathrm{amp}$. per sq. $\mathrm{dcm}$. This accounts for the fact that its occurrence was not observed by McElfresh, ${ }^{1} \mathrm{~F}$. Fischer, ${ }^{2}$ and other earlier experimenters upon the change of the electrical resistance of palladium with cathodic occlusion of hydrogen. Examination of Fischer's data shows that in certain of his experiments, in which the current density was somewhat higher than usual, he did in fact detect this change of resistance after the interruption of electrolysis, although unaware of its nature. Thus with a wire of $0.3 \mathrm{~mm}$. diameter, and a current strength

i Proc. Am. Acad., 39, 323 (1904).

2 Dissertaiion, Leipzig, 1906; Ann. Phys., [4] 20, 503 (1906). 
of 20 milliamp., he found a gain in resistance of $2.5 \%$ after the charging circuit was broken. The length of this wire was not given, but upon the assumption that it had a length approximately the same as those used in his other experiments, the mean length of 5 such wires being $227 \mathrm{~mm}$., the current density would be 0.93 amp. per sq. dcm. Fischer attributed this phenomenon to the gradual diffusion of the hydrogen into the interior of the wire, and the consequent increase in the amount of alloy, after the cessation of electrolysis. This explanation, which would suffice for his own observation taken alone, is obviously inadequate to explain various observations of Martin's and of the present study, of which those shown in Fig. $4 b$ may be especially mentioned.

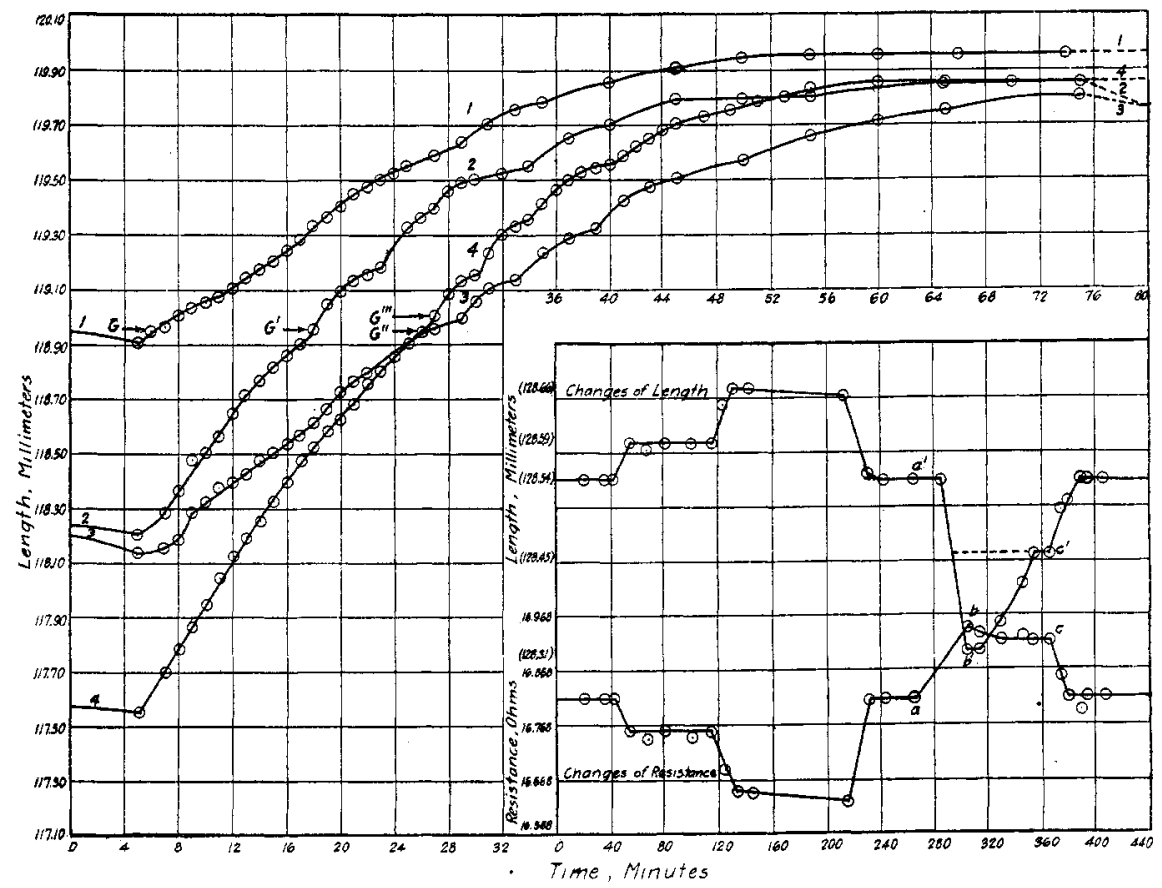

Fig. 4a.-Changes of length of "Renewal Curve." G, G', G" and G'"

Fig. $4 b$. - Inverse relation of resistance correspond to the same points in Fig. 6.

The current density employed determines also the rapidity of the supplementary rise after interruption. This may be seen from the curves in Fig. 5, where the values were approximately: 1.26 amp. per sq. dcm. for curve No. $1 ; 0.63$ for Nos. 2 and 3 ; and 0.23 for No. 4 . Thus it will be seen that while the maximum value of the resistance is itself very little affected, the rapidity with which it is reached depends upon the current density. Moreover, with approximately the same current density, the 
slope of the curve can be very closely reproduced as is shown by curves Nos. 2 and 3 , in Fig. 5.

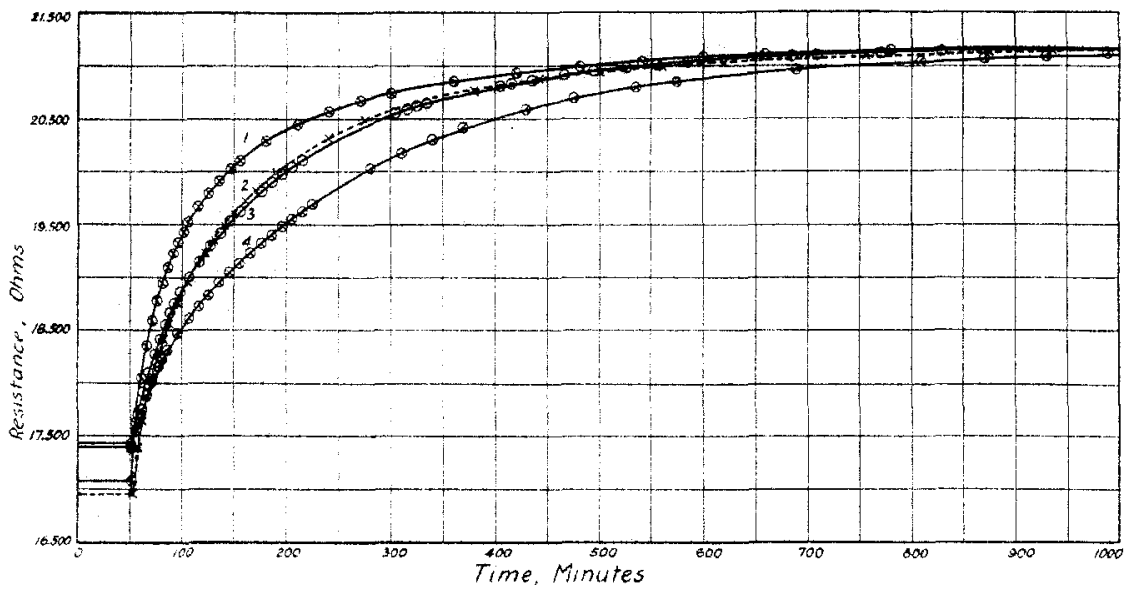

Fig. 5.-Evolution curves.

The supplementary gain in resistance (evolution curve) is observed not only when electrolysis is discontinued after a steady resistance has been reached, as is shown by the branch $\mathrm{C} \mathrm{D} \mathrm{E}$, Fig. 2, but it may also be obtained by interruption upon any point of the charging curve, as is represented by $\mathrm{MNO}$ and $\mathrm{PQR}$ of the same figure. The curves just mentioned, unlike the rest of Fig. 2, are plotted from the actual results of two successive experiments upon a $0.05 \mathrm{~mm}$. wire, and are not merely ideal curves typical of the entire body of observations.

\section{A Possible Explanation.}

All of the results which have been discussed are most simply explained upon the conception tentatively advanced to account for the observations of Martin. According to this, it was supposed that the hydrogen, upon first entering a palladium cathode, is in a transitional state, in which it increases the conductance of palladium; or, in other words, that the hydrogen at this stage has at least an apparent conductance of its own and that this transitional form gradually passes into another which has the property of diminishing the conductance of the metal. In the second of these forms it is natural to suppose that the hydrogen and palladium are combined to produce an alloy of the solid solution type, since this would make the conduct of the hydrogen entirely analogous to that shown by substances in general, when dissolved in solid metals. As regards the first, or transitional form, which has an apparent conductance of its own, it is possible that this consists simply of hydrogen ions, driven into the interior of the metal by the potential gradient, and not yet discharged. Upon this point, however, it will be well to avoid any definite assump- 
tion. The supposition of two forms, possessing the properties indicated, would account adequately for all the influences of current density which have been discussed.

The higher the current density the more rapid is the production of the conducting hydrogen within the metal. With very high current densities the diminution of resistance, due to this form, would, therefore, be particularly in evidence, while the increase in resistance, due to the production of the second (alloy?) form, would take place gradually. The joint result of these two effects would constitute the observed resistance.

With a high current density of approximately I. 50 amp. per sq. dcm. the charging curve has the form shown in No. I of Fig. 3. Here it may be supposed that in the early stages of occlusion alloy formation occurs very rapidly, while its rate perhaps diminishes as the relatively slow processes of diffusion come more and more into play. Moreover, from analogy with all known alloys of the solid solution type, it is probable that the first portions of hydrogen produce a relatively large increase of resistance, while later additions have a much smaller effect. As a consequence of both of these factors, the increase of resistance resulting from formation of the alloy is at first very rapid. Since, in this early stage the consumption of the transitional form is also rapid, the decrease of resistance due to the accumulation of the conducting hydrogen is slow. The observed resistance, therefore, being the composite effect of a rapid increase and a slow decrease of resistance, appears as the rapid rise constituting the first branch of the curve. As the alloy formation slackens, the accumulation of conducting hydrogen becomes more rapid and its effect in diminishing resistance grows more pronounced. 'Since, at the same time, the opposite effect of the alloy formation is decreasing for the two-fold reason already mentioned, there comes a time when the effect of the accumulating conducting hydrogen overcomes that of the alloy formation. At this point the observed resistance passes through its maximum. The relative minimum of the observed curve at the point $X$ may be supposed to be due to the attainment of a maximum concentration of conducting hydrogen, determined by the current density, before the alloy formation has wholly completed itself, so that the effect of the latter again comes into evidence in the last portion of the charging curve.

With lower current densities the alloy formation is less rapid, the experimental curve rises less steeply, and shows a less pronounced maximum. This form of curve, the type of which is shown in Fig. 3, No. 3, and in the typical Fig. 2, is obtained with current densities in the neighborhood of 1 . IO amp. per sq. dcm. With very low current densities, below about $0.3 \mathrm{amp}$. per sq. dcm., the rise in the observed resistance is still more gradual, and continues regularly to the steady value without exhibiting any perceptible maximum. 
In a similar manner, the evolution curve may be regarded as the composite result of a gain of resistance due to the continued conversion of conducting hydrogen into alloy hydrogen, and the loss of resistance due to the breakdown of the alloy with the evolution of gas. The latter of these effects is not evident unless oxygen has access to the palladium. For in experiments conducted in a closed cell, with an electrolyte saturated with hydrogen, as in the experiments of Martin, and in some preliminary experiments of the present investigation, the evolution curve, after reaching an approximate maximum (D, Fig. 2), continues horizontally, or, rises very gradually, as is indicated by the dotted lines $D d$ and $D e$. In the ordinary experiment with the open cell, however, the evolution curve rapidly falls away from its maximum, as is indicated by the line D E F.

The effect of the current density at which the steady value of resistance $\mathrm{C}$-is reached, may also be explained upon the suppositions just discussed. For at the steady value, the composition of the alloy, which is then saturated, probably varies very little, if at all, with the current, while the quantity of conducting hydrogen increases greatly with the current density. Hence, when the interruption is made at a high current density, the large amount of conducting hydrogen escapes quickly, producing a rapid rise in the observed resistance.

It is of course not meant to be inferred that the above conception is the only one possible, but merely that it seems to afford the simplest explanation of all the facts as yet brought to light. That the hydrogen present in the first stage of occlusion consists of one or more forms in a condition different from that generally exhibited by a component of a metallic solid solution is evident from its transitional character and from the fact that its effect upon the conductivity of the occluding metal is the opposite of that which is invariably produced by the formation of such solutions. The second form of hydrogen, on the other hand, appears to comport itself in the manner to be expected of an element forming a solid solution with the occluding metal. Its disappearance, as well as its formation, is a relatively slow process, and the latter is attended by a marked decrease in the conductivity of the metallic solvent. The question as to whether a compound is formed is but one phase of the broad problem regarding the constitution of crystalline solutions, and may here be ignored. The terms "conducting hydrogen" and "alloy hydrogen" seem suited to express what appears to be already established, without implying assumptions which are at present without purpose.

\section{Deductions and Their Confirmation.}

Upon the conception just discussed, it is possible to predict in general terms the behavior of palladium under quite different electrolytic treatment. 
In the first place, if the electrolytic evolution of hydrogen upon the palladium be renewed after a portion of the occluded hydrogen has escaped, it is to be expected that the changes of resistance which will result will depend upon the degree to which the alloy has broken down.

If electrolysis is renewed just when the maximum of the evolution curve has been reached and when, as has been supposed, the conducting hydrogen has practically all escaped, while the alloy is still very nearly saturated, the only effect will be to restore the lost conducting hydrogen, and, hence, to diminish the observed resistance. If, on the other hand, electrolysis is not renewed until the evolution has continued past the maximum of the curve, so that a portion of the alloyed hydrogen has es-

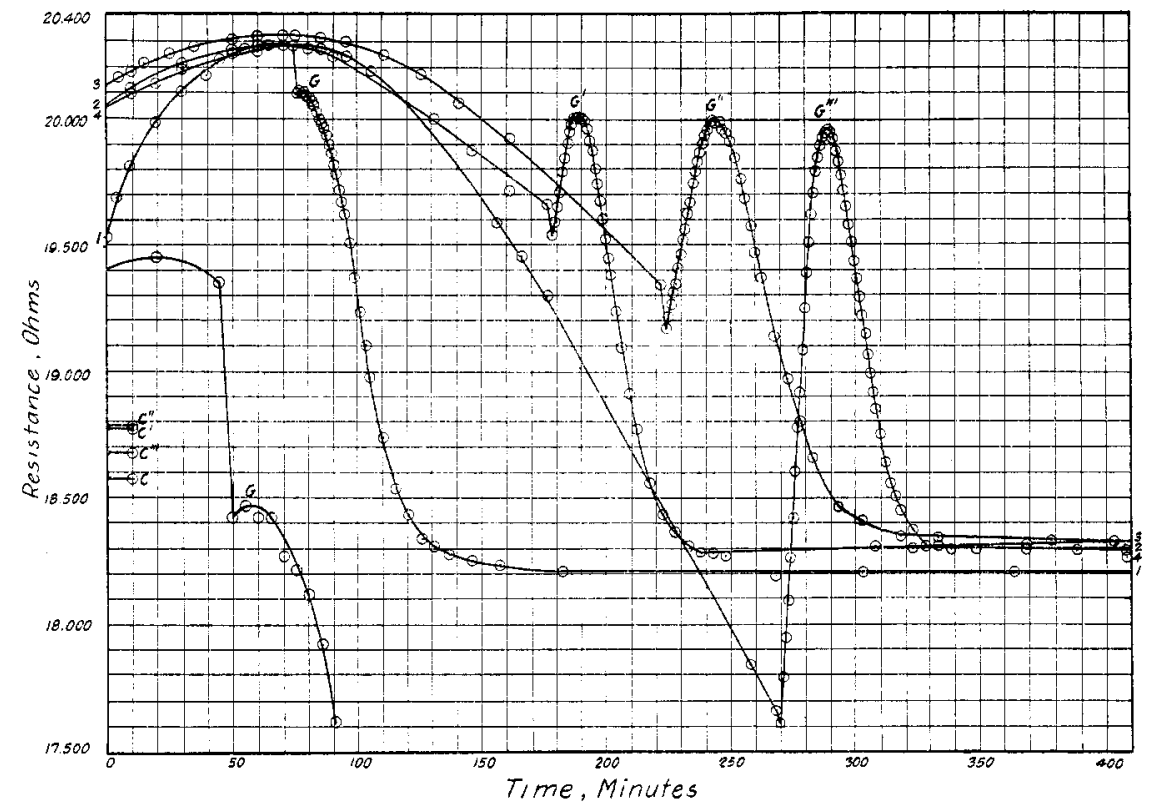

Fig. 6.-Renewal curves.

caped, the effect will be more like that produced on first charging the empty wire. The observed resistance will, therefore, rise to a maximum while the alloy is being restored, and afterward decline as conducting hydrogen accumulates.

This conduct was actually found in the experiments the results of which are displayed in Fig. 6. It will be seen from these curves that the rise of resistance, due supposedly to the renewed formation of alloy, becomes greater as the renewal of electrolysis is begun at points lower and lower upon the falling branch of the evolution curve.

There are two characteristics of these curves which were not foreseen, and which app ear to be illuminating. In the first place, it will be noticed 
that the expected rise of resistance, due to alloy formation, was preceded by a small, sudden drop. This drop appears to be greatest when the renewal is made at the maximum of the evolution curve, as in Curve No. I, Fig. 6, which seems to indicate that a certain accumulation of conducting hydrogen occurs before the formation of alloy makes itself evident. It may be supposed that the transitional form must reach a certain concentration, determined by the composition of the partly broken down alloy, before alloy formation again begins.

In the case of an alloy which is almost wholly broken down (Curve No. 4, Fig. 6), this effect is scarcely apparent. In the case of new empty wires, where electrolysis is not renewed, but begun for the first time, the numerous experiments which have been made show no indication of this initial drop, except in wires which have been previously annealed in air as will be explained later.

The second point in connection with these curves, is the very regular way in which the maxima decrease. The four maxima $G, G^{\prime}, G^{\prime \prime}$, and $\mathrm{G}^{\prime \prime \prime}$, lie very nearly upon a straight line. This accords well with the conception that the observed curves are the result of two superimposed effects, the second of which (accumulation of conducting hydrogen) has greatest opportunity to exert its influence in the cases in which the alloy has broken down to the greatest degree, and the time elapsing before the attainment of the maximum is consequently longest.

The small dip in the last portion of the renewal curve shown at $\mathrm{H}$, in Fig. 2, appears to be due entirely to a lag in the expansion of the wire, since it disappears when resistivities instead of resistances are plotted.

The second prediction to which the above-mentioned conception of the occlusion processes leads is in respect to the conduct of a charged wire when the direction of the electrolytic current is reversed. Under these circumstances it might be expected that the conducting hydrogen would be removed more rapidly than the alloy hydrogen, producing in consequence a rise of resistance, followed by a decline after the conducting hydrogen had been largely exhausted, so that the effect of the removal of alloy hydrogen became evident. This expectation also is fulfilled, as is shown by the results of experiment in Fig. 7. Each of these curves corresponds to an experiment in which the current was reversed after a steady resistance had been reached. The steady value of resistance obtained by the evolution of oxygen is very slightly higher than the original resistance of the wire, and this difference is not wholly accounted for by the slight lengthening of the wire which has taken place. If the resistivities at the beginning and end of the experiment are calculated upon the assumption that the diameter of the wire remains unaltered, there is found an apparent increase of resistivity of $0.5 \%$. This difference is very small, in view of the fact that between the two measurements just 
referred to, the wire had been subjected to the entire series of operations represented by the curves of Fig. 2 .

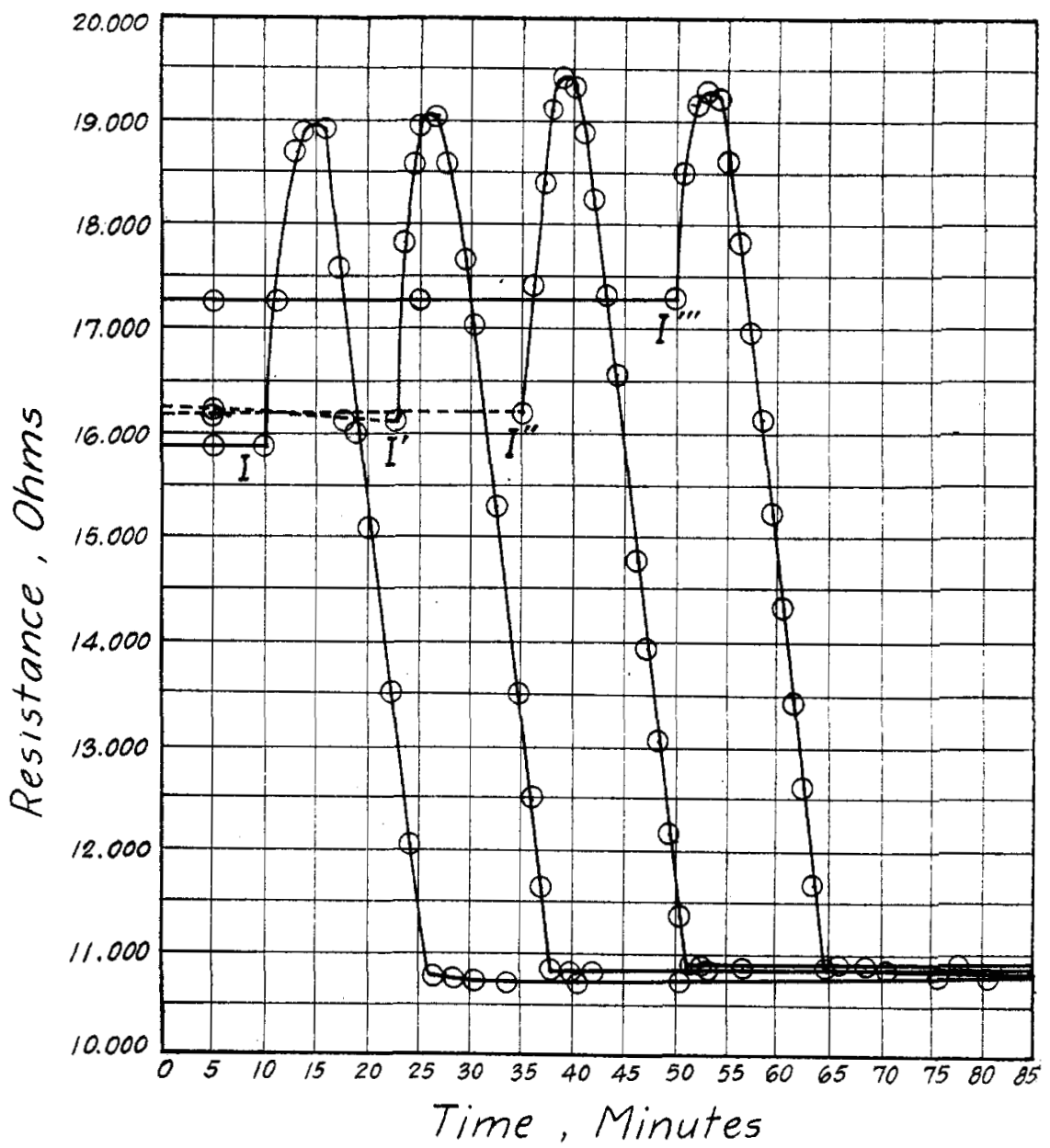

Fig. 7.-Discharge curves.

\section{The Influence of Oxygen.}

In order to make sure whether the changes of resistance obtained by reversal of electrolysis were entirely due to removal of hydrogen, or whether the oxygen itself affected the resistance of the palladium, experiments were made in which new wires, not previously charged with hydrogen, were subjected to anodic treatment. The wires were annealed in nitrogen, in the manner which has already been described, and in order to make possible the measurements of the changes of resistance, which were shown by a preliminary experiment to be very small, a more sensitive method of measurement was substituted for that previously 
used. To this end, the resistance of the wire after it had been annealed in the cell was determined as usual with the Kelvin bridge. This gave the actual resistance of the wire, not including that of the connecting leads. A Callendar and Griffiths' bridge, intended for resistance thermometry, was then substituted for the Kelvin connections, and a piece of manganin wire, in the compensating arm of the new bridge, was adjusted until the correct reading of resistance, known from the preceding measurement, was reproduced. It was thus possible to measure the relative resistances of the wire, at different stages in a given experiment, to about two parts in one hundred thousand. This refers only to measurements on palladium, in all of which the wires were of $0.05 \mathrm{~mm}$. diameter.

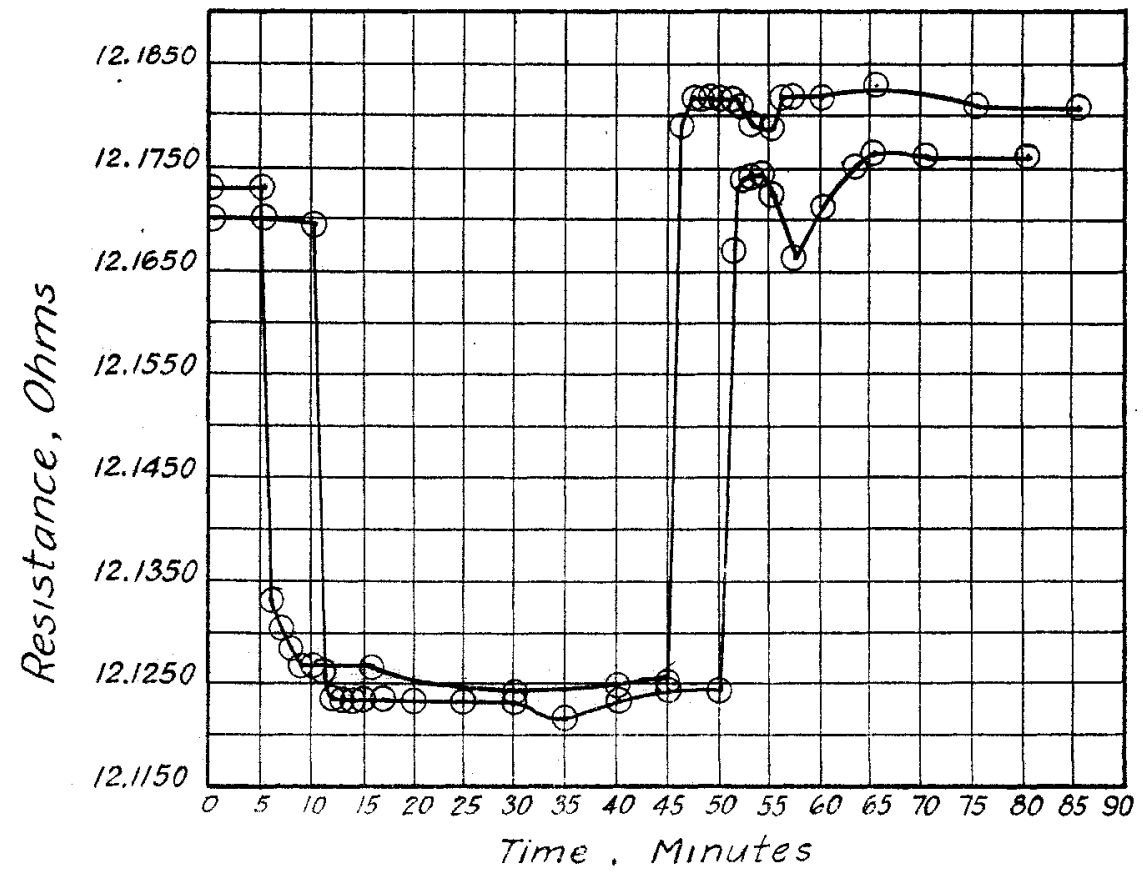

Fig. 8.--Influence of oxygen upon resistance of palladium.

On charging the wire with oxygen the resistance immediately fell, reaching a steady value. Upon the interruption of electrolysis, it instantly rose and again became steady at a value somewhat higher than that which it originally possessed. The electrolysis was then renewed, with similar results. The figures from 4 repetitions with the same wire are shown in Table II, and the second and third of these experiments are represented in Fig. 8.

For a given current density, the depression produced by oxygen is much less than that given by hydrogen. Thus in experiments in which the current density was approximately $\mathrm{I}$.oo amp. per sq. dcm., the re- 
sistance of the palladium wire was diminished, in the case of hydrogen, by from 25 to $33 \%$ of the original value, while oxygen caused depressions of only $0.3 \%$, in each of the two experiments made under these conditions. The hydrogen effect at this current density was, therefore, about roo times as great as that produced by oxygen.

TABLE II.

$\begin{array}{lccll}\text { Expt. } & \begin{array}{c}\text { C. D. } \\ \text { amp./dcm }\end{array} & \begin{array}{c}\text { Original } \\ \text { resistance. }\end{array} & \begin{array}{l}\text { Steady } \\ \text { vaiue. }\end{array} & \begin{array}{l}\text { Final } \\ \text { value. }\end{array} \\ \text { C 6,I } & \text { I .OI } & \text { I2.165 } & \text { I2.I55 } & \text { I2.I7O } \\ \text { C 6,2 } & 0.88 & \text { I2.I695 } & \text { I2.I245 } & \text { I2.I730 } \\ \text { C 6,3 } & 0.88 & 12.1730 & \text { I2.I250 } & \text { I2.I8I4 } \\ \text { C 6,4 } & \text { I.OI } & 12.1814 & \text { I2.I435 } & \text { I2.I92 }\end{array}$

As will be seen from this table, the changes are of small magnitude. They show, however, that the effect of oxygen is similar to that which would be produced by conducting hydrogen, if there were no formation of hydrogen alloy. This result is striking, as it seems to indicate that oxygen also enters the metal in a transitional form possessing conductivity of its own. In addition the oxygen seems to have an irreversible effect upon the metal, which raises the resistance of the latter during each treatment, by amounts which varied in these experiments from 0.029 to $0.087 \%$. This gain is probably the result of the formation of an oxide, as will be shown in the next paragraph. The smallness of the effects shows that the oxygen cannot be directly responsible for the changes exhibited in Fig. 7, which must, therefore, have been due to the removal of hydrogen.

It seemed interesting to examine the conduct of a wire, which had thus been altered by treatment with oxygen, upon being charged in the ordinary way with hydrogen. At the end of the experiments, the results of which have been shown in Table II, the direction of the current was therefore reversed. The hydrogen charging curve obtained showed no noticeable difference from all the others given by similar current densities, except that there was an initial drop in the resistance of $0.8 \mathrm{I} 2 \mathrm{ohm}$ before the beginning of the usual rise. This could not be sufficiently accounted for merely from the reduction of the supposed film of oxide, since, as may be seen from Table II, this had produced a total increase of resistance of only $0.027 \mathrm{ohm}$. The large initial drop may readily be explained, however, by supposing that the presence of a film of oxide prevented the immediate formation of hydrogen alloy, so that in the early stages of the experiment the effect of the accumulation of conducting hydrogen was evident in the fall of resistance.

In order to see whether the influence of oxygen could be intensified, several experiments were next made in which a wire was anodically freed from hydrogen and then annealed in air, instead of nitrogen, before subjection to the usual hydrogen treatment. In these cases the charging 
curve exhibited an initial drop, similar to that obtained after anodic polarization, but more pronounced. In one instance, in which the current density was practically the same as in the experiments just described, the resistance fell $\mathrm{I} .355 \mathrm{ohms}$, and remained for about three minutes almost constant before the charging curve began to rise.

In addition, another effect was obtained which was not produced merely by anodic polarization. This consisted in a marked elevation of the steady value I, Fig. 2. This elevation is particularly well shown by Fig. 7 , where curve No. I shows at I a steady value obtained before a wire had been subjected to oxygen; curves Nos. 2 and 3 give the values $I^{\prime}$ and I" after two successive anodic polarizations of the same wire; and curve No. 4 exhibits at $I^{\prime \prime \prime}$ the effect of a subsequent annealing in air.

The fact that the influence of severe oxidation persists, even after the wire has been saturated with hydrogen to constant resistance, is most simply accounted for by supposing that the successive formation and reduction of an oxide has produced a certain amount of finely divided metal, thus increasing the effective surface and causing results similar to those which would follow from diminishing the current density upon which the magnitude of the steady value depends. It may be remarked that a repetition of the annealing in air produced only a very slight further elevation of the steady value. An increase of the effective surface would also account for the fact repeatedly observed, that oxygen treatment renders a wire more readily susceptible to the action of hydrogen. Wires previously oxidized pass much more rapidly through all of the phases of the occlusion of hydrogen.

\section{Changes of Length.}

In all of the experiments hitherto discussed, observations of the changes of length of the wire were made simultaneously with those upon the alterations in its resistance.

During the processes represented by the evolution and discharge curves, or in other words, while hydrogen was being lost from the wire, the length diminished at a rate which varied slightly during the loss of the first portions, but which thereafter became so nearly constant that no irregularities could be detected although the measurements were sufficiently accurate to show a change of less than two parts in ten thousand.

On the other hand, the elongations which occurred during the occlusion of hydrogen, either on first charging or on renewal of electrolysis, exhibited fluctuations which appear to be instructive and which are shown in curve No. 6 of Fig. 3 and in the curves of Fig. 4a. These fluctuations show that the stretching of the wire was discontinuous. Exactly similar conduct is shown when wires are stretched by the application of external forces, and the curves to which reference has just been made bear a striking 
resemblance to those obtained by de Forest ${ }^{1}$ in studies of the plastic elongation of brass and bronze wires. This seems to indicate that the enlargement of the palladium during the occlusion of hydrogen is due to an expansive force exerted by the latter element, rather than to a crystalline rearrangement or other similar change accompanying the alloy formation.

The most important result, however, obtained from the measurements of length is not the manner in which the elongation varies during the charging or discharging of the wire, but the relation between the steady values of length reached with prolonged electrolysis and the corresponding steady value of resistance. This relation is shown in Fig. $4 b$.

As has already been explained, a different steady value of resistance was obtained for every different current density. The curves of Fig. $4 b$ represent the simultaneous variations of length and of resistance produced by varying the charging current. This experiment was performed with a wire of the carefully purified palladium $P$, of $0.05 \mathrm{~mm}$. diameter. As will be seen, every new value of current density produced changes in the steady values of resistance and of length, which were in opposite senses. This inverse correspondence between resistance and length affords one of the best supports for assuming the existence of two forms of hydrogen. Moreover, since the alloy is doubtless saturated throughout all of the changes represented in this figure, the conclusion seems to be indicated that the variations of length are due to the changes in the quantity of conducting hydrogen.

In this connection the forms of the two curves of Fig. $4^{b}$ at the corresponding points of the region $a b c$ are instructive. When the applied voltage was decreased at $a$, the diminution of current evidently produced a falling off in the quantity of conducting hydrogen, which was responsible for the shortening of the wire seen at $a^{\prime} b^{\prime}$ and the increase of resistance observed at $a b$. The loss of conducting hydrogen appears, however, to have overshot the equilibrium, producing the sag in the length curve at $b^{\prime}$; and to this there corresponded a similar, but of course inverted, change in the resistance, seen in the small maximum at $b$. Similar maxima were several times observed by Martin. ${ }^{2}$

From this it appears that the expansion of the palladium which occurs during cathodic occlusion is in very large part due to the presence of the transitional form of hydrogen in the metal. This accords with the indication obtained above from the form of the curves in Fig. $4 a$, that the stretching occurs as the result of an expansive force exerted by the hydrogen. So far, then, as the results of the present experiments are concerned the volume changes might be attributed wholly to this influence of the conducting

1 Proc. Am. Soc. Testing Materials, 18, II (I916).

${ }^{2}$ Loc. cit., Fig. 3, and p. 2588, lines 14 and 15 from bottom. 
hydrogen, and there is no indication that the alloy formation is responsible for any of the changes of dimensions. Such a conclusion would, however, seem to accord poorly with the fact that palladium also expands when it occludes hydrogen from the gas phase. That the greater part of the change of volume is due to the unalloyed hydrogen may, however, be deduced from certain experiments of Thoma ${ }^{1}$ and of F. Fischer. ${ }^{2}$ Both of these experimenters observed that the elongation during cathodic occlusion is proportionally greater for the last portions of the gas taken up than for earlier fractions. Since the alloy is undoubtedly saturated before these last portions are occluded, this "supersaturation hydrogen" goes wholly to increase the quantity of conducting hydrogen, if the conception as to the existence of the two forms is correct. Hence the last portions of the occluded gas would produce, in greater degree than those taken up during the earlier stages of occlusion, any effect which is due, wholly or chiefly, to the presence of conducting hydrogen.

The predominant role of the conducting hydrogen in determining the volume of the wire is also shown by some of the present experiments, in which the hydrogen was allowed to escape spontaneously from a wire which had been saturated. Under these circumstances the wire did not return to approximately its original length, as has been shown to be the case when the hydrogen was expelled by careful annealing, but instead the wire contracted until it was shorter than before the occlusion; that is to say, it exhibited what Graham ${ }^{3}$ termed "retraction." In these cases the original length was again reached while the resistance was still considerably greater than that of the empty wire. In one such experiment it was approximately $40.0 \%$ greater than the original when the elongation had fallen to zero. Thus the elongation had completely disappeared while much of the alloy still remained.

As was to be expected from the smallness of the resistance changes produced, the evolution of oxygen upon palladium wires not previously charged with hydrogen caused no measurable alterations of length.

\section{Conduct of Some Other Metals.}

From the conceptions as to the occlusion of hydrogen by palladium which have been set forth in the preceding pages, it was to be expected that metals such as tantalum and iron, which are known to occlude hydrogen in considerable quantity, would exhibit changes of resistance altogether analogous to those shown by palladium; and that a metal such as platinum, which is known to occlude very little hydrogen, would exhibit only the effects which have been attributed to "conducting hydrogen."

In order to test the correctness of these predictions a few experiments

Z. physik. Chem., 3, 69 (1889).

2 Dissertation, Leipzig, 1906; Ann. Phys., [4] 20, 503 (1916).

3 Proc. Roy. Soc. (London), 17,212 (1869). 
were made in which changes of length were not observed, and in which the apparatus shown in Fig. 9 was employed. The figure represents only the cathode vessel, which connected through the tubes $c$ and $c^{\prime}$ with an anode vessel of similar form. The experiment wire was gold-soldered to the platinum point $b$, and to the gold point $b^{\prime}$, upon the rotating stirrer. Electrical connections were made through two mercury cups, one of which served to exclude the air from the apparatus. The electrolyte employed was $2 N$ sulfuric acid, as in the experiments already described. During the course of each experiment the stirrer was kept in motion at the rate of about $45 \mathrm{r}$. p. m. The electrical resistance was observed with the Callendar and Griffiths bridge already mentioned. The metals investigated were not annealed, except in the case of platinum. In this experiment a glass bell was substituted for the cell chamber containing the electrolyte and an atmosphere of nitrogen was maintained about the cathode wire, while the annealing was conducted in the usual manner.

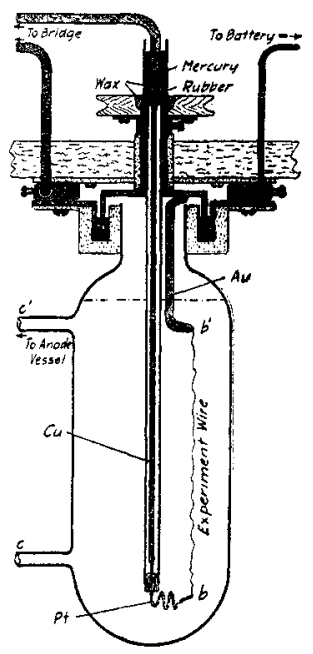

Fig. 9.-Cathode vessel.

\section{Tantalum.}

Tantalum wires of 90.0 to $91.0 \mathrm{~mm}$. in length, and $0.1 \mathrm{~mm}$. in diameter were used. Owing to the extreme readiness with which tantalum reacts both with nitrogen and oxygen, great care was necessary in attaching these wires. The ends were first covered with a thin film of molten borax. This prevented the wire from coming into contact with the atmosphere while it was thrust into a small molten globule of purified gold. The gold was then hammered into a thin spade and fused to the rotating stirrer. Whatever borax remained on the wire after the completion of this operation was removed by solution.

The current strength was varied between about 7.0 and 15.0 milliamp., corresponding to a current density of from 0.27 to 0.55 amp. per sq. dcm. The current density was rendered much smaller than the current seems to indicate because of the excess cathode area exposed by the platinum and gold wire connections.

In the early stages of electrolysis, a fall of resistance was observed, similar to that obtained with palladium which had been subjected to strong oxidation. In view of the comparative readiness with which tantalum oxidizes this is not surprising. The initial drop was followed by a steady rise of resistance which continued until the wire broke, which in one case was not until a gain of $20.7 \%$ over the original resistance had been reached. To determine whether the expected supplementary rise (evolution curve) occurred, it was necessary to interrupt at an early stage of the electrolysis. 
This caused a steady rise of resistance, apparently wholly like that found with palladium, which amounted in one case to $2.9 \%$. It may, therefore, be said with certainty that tantalum exhibits the expected behavior.

Iron.

Wires of very soft iron, intended originally for magnetic experiments, having a length of $80 \mathrm{~mm}$. and a diameter of $0.135 \mathrm{~mm}$., were employed. A current density of approximately I.44 amp. per sq. dcm. was used, and the wire was polarized before allowing the electrolyte to enter the cell in order to obviate the solvent action of the sulfuric acid. An initial drop of the resistance was observed as in the case of tantalum, and this was followed by an increase, which in one instance reached $5.8 \%$ above the original value. At this point the wire broke. A supplementary gain after the interruption of electrolysis could not be sought because of the solubility of iron in the electrolyte, but by varying the current density it was shown that the resistance varied in the opposite sense, as in the case of palladium. The curves obtained in the case of iron were slightly irregular, due doubtless to the lack of annealing. There seems to be no doubt, however, that iron also behaves like tantalum and palladium.

\section{Platinum.}

The platinum wire used was $97.0 \mathrm{~mm}$. in length and $0.05 \mathrm{~mm}$. in diameter. It showed an initial resistance of $4.9183 \mathrm{ohms}$, as compared with 4.9174 ohms calculated for pure platinum from the data of Jaeger and Diesselhorst. ${ }^{1}$ The current density was varied from about 0 . Io to 0.36 amp. per sq. dcm. Upon application of the current, the resistance fell to a steady value, and when the current was increased, the resistance went still lower, to a new steady value. Upon the interruption of electrolysis, the resistance gradually rose to that originally possessed by the wire. The wire was then annealed in the usual manner and the experiment repeated. It behaved as has just been described, except that the fall in resistance was more regular, due in all probability to the fact that the wire was not annealed in the first experiment. Thus platinum showed the behavior which had been expected for a metal which occludes very little hydrogen, except for the extreme slowness of the recovery after interruption. It would be premature to draw any further conclusions from the results of these two experiments, but it may at least be suggested that the loss of resistance would be very slow in the case of a metal exhibiting little alloy formation if the production of this alloy were a necessary intermediate step in the conversion of conducting hydrogen into the gaseous form in which the element escapes.

\section{Conclusion and Summary.}

The outcome of these experiments has shown that the conduct of palladium, tantalum, iron and platinum, under widely varied conditions of

Wiss, Abh. Reichsanstill, 3, 269 (1900). 
electrolysis, accords, at least qualitatively, with a conception reached from an earlier study of palladium. This conception is that hydrogen when evolved electrolytically enters the metal of the electrode in a transitional form, which produces a diminution of the resistance of the metal; and this transitional form, in the cases in which the gas in question is largely occluded, passes gradually into another, which has the opposite effect upon the resistance. Indications have also been obtained that the conduct of oxygen is similar to that of hydrogen.

The quantity of the transitional form present when continued electrolysis has led to a steady state of resistance, is dependent upon the current density. In the case of hydrogen, at least, it may reach very high values. The consequent diminution of electrical resistance is also large in the case of hydrogen, but much smaller in that of oxygen, when the current densities are the same.

The transitional form of hydrogen appears to be responsible for the major part of the changes of dimensions produced in palladium by electrolytic occlusion.

In the more persistent, or "alloy" form, the hydrogen is firmly held by palladium up to a critical temperature which has not been determined, but which lies probably not far from $300^{\circ}$. At this temperature hydrogen begins to be evolved rapidly.

The foregoing observations are of interest because of the important role in the mechanism of the electrode processes, which they seem to make it necessary to assign to reactions occurring within the electrode.

[CONTRIbutron From the Department of ChEMistry of CORNELI, University.]

\section{GALLIUM. ${ }^{1}$}

By L. M. Dennis and J. Allington BridgMan. Received July 26, 1918.

This article deals with the spectroscopic detection of gallium, indium, and zinc; the purification of gallium; the determination of gallium, and of gallium, indium, zinc, and aluminum in the presence of one another; and the preparation and properties of two new salts of gallium, and the properties of some salts that had previously been prepared.

\section{Material.}

The crude material employed in this investigation consisted of about $35 \mathrm{~g}$. of an alloy consisting chiefly of gallium and indium, which was generously presented to us by Mr. Kurt Stock, Superintendent of the Bartlesville

1 This article is based upon the thesis presented to the Faculty of the Graduate School of Cornell University by J. Allington Bridgman in partial fulfillment of the requirements for the degree of Doctor of Philosophy. 